\section{El valor del proceso como metodología proyectual}

The Value of the Process as a Project Methodology

Dionisio Sánchez Rubio

Palabras clave: Metodología, Diseño Social, Diseño Crítico, Diseño especulativo Key words: Methodology, Social Design, Critical Design, Speculative Design
Las metodologías proyectuales surgidas durante la consolidación del diseño como disciplina ya no son válidas en el contexto actual, ahora vivimos en una sociedad mucho más contexto actual, ahora vivimos en una sociedad mucho más compleja y los problemas que surgen de esta requieren de nuevos métodos de trabajo para resolverlos. Hoy en día es mu difícil plantear soluciones de diseño a problemas concretos. Las diferentes problemáticas sociales surgen de situaciones inciertas, tan inciertas y maleables como la sociedad en la que vivimos. Por lo tanto, nuestra mirada como diseñadores debe practicarse desde la lente del investigador, que se acerca a la

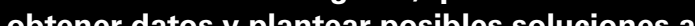
problemas reales.

The project methodologies that emerged when design was becoming established as a discipline are no longer valid in the current context. We now live in a much more complex society and solving the problems arising from it requires new working methods. Nowadays it is very difficult to suggest design solutions to specific problems. The different social problems arise from uncertin situations. The different social problems arise from live in. As a rions, as uncertain and malleable as the society we ive in. As a result, as designers, we must look through the lens of the researcher, approaching society to obtain data and suggest possible solutions to real problems.

\section{El valor del proceso como metodología proyectual}

Las metodologías del diseño han evolucionado a lo largo de la historia debido a la necesidad de organizar y controlar los procesos cada vez más complejos en la fabricación de los objetos. "La expresión metodología del diseño, como el diseño mismo, abarca un conjunto de disciplinas en las que lo fundamental es la concepción y el desarrollo de proyectos que permitan prever cómo tendrán que ser las cosas e idear los instrumentos adecuados a los objetivos preestablecidos" Hay que destacar que metodología y método suelen ser confundidos o utilizados como sinónimos. Mientras que los métodos del diseño son instrumentos que nos permiten cumplir con nuestros objetivos en el proceso de diseño, la metodología se encarga del "estudio o reflexión de los métodos, al análisis del conjunto de procesos y métodos que se siguen en la proyectación de diferentes productos"

Los métodos utilizados en el diseño tienen un carácter retrospectivo, es decir, se basan en experiencias previas para dar solución a problemas de diseño. Éstos se combinan y modifican en función de las nuevas técnicas de producción, viéndose condicionados por los avances tecnológicos. Este desarrollo método-tecnología se ve coartado por la experiencia previa y muchas veces no tiene en cuenta el contexto sociocultural en el que opera el diseño. Además, los métodos del diseño tienden a ordenar y buscar un orden lógico en la solución de un problema a través de una secuencia lineal en su desarrollo. Si los métodos del diseño tienden a estructurar la información para encontrar un orden racional en la solución de un problema de diseño ¿Cómo se puede dar solución a los problemas planteados por una sociedad repleta de contradicciones?

La evolución de los métodos ha sido progresiva y su finalidad consiste en reducir la complejidad por medio de la racionalidad, pero ¿qué sentido tiene basarse en los métodos tradicionales del diseño en una sociedad cada vez más compleja?. “La perspectiva que ofrece mirar el diseño desde el pensamiento complejo permite dilucidar que no hay recetas para obtener los resultados esperados de diseño, ya que este último es un sistema en constante cambio" ${ }^{\prime 3}$. Los diseñadores están investigando profundamente en otras disciplinas seleccionando determinadas metodologías, tecnologías y enfoques desde donde poder modificar su programa, y al mismo tiempo, trabajar en el desarrollo y la creación de redes con otras disciplinas propiciando un cambio en el modo de entender el diseño, desplazándolo de algo percibido como independiente e individual, a algo que se percibe en términos de su relación con la sociedad.

\section{Constantes metodológicas}

Los estudios en relación a las metodologías proyectuales demuestran que existen determinadas fases que aparecen como constantes en los procesos de diseño. El proceso de diseñar se inicia con la recogida de información perteneciente al problema a resolver. La información se estructura de forma lógica para entender mejor el problema y de su interpretación obtenemos los requerimientos y condicionantes del proyecto. Una vez 
analizados se generan propuestas acordes a las exigencias del mismo, para posteriormente crear una propuesta formal para su producción.

Los métodos del diseño se han visto influenciados por los métodos técnico-científicos de otras disciplinas como la teoría de sistemas, la teoría de la información y la aparición de la informática. Las técnicas creativas del brainstorming y la sinéctica, han sido cruciales en el desarrollo de las metodologías proyectuales en las últimas décadas del siglo XX. A lo largo de su evolución podemos detectar tres momentos clave: el primero se produce cuando empiezan a tomarse en consideración los procesos del subconsciente del diseñador como procesos imposibles de explicar con el método científico; el segundo coincide con el período posmoderno, momento en el que se rompe con los métodos propuestos por el funcionalismo, y el último cuando se empieza a hacer especial hincapié en las ciencias filosóficas, donde adquiere relevancia el contexto y se pone en valor la necesidad de diseñar de manera transdisciplinar.

Los métodos del diseño y las metodologías que las agrupan ya no son válidos para resolver problemas que se derivan de la complejidad social. Ante esta situación es pertinente crear un marco de reflexión que nos permita abordar otras propuestas metodológicas a través del análisis de proyectos cuyos planteamientos se escapan de la lógica metodológica tradicional.

\section{El valor del proceso: el diseño como medio}

En la última década han surgido metodologías proyectuales basadas en el proceso. Un claro ejemplo lo encontramos en el proyecto Agile 4 que se define "como un marco de trabajo basado en un manifiesto publicado en el año 2001 cuyos principios son el valor del work in progress, la responsabilidad de cada individuo durante el desarrollo y una actitud positiva de respuesta frente a los cambios" ${ }^{5}$. Tal y como señala Rosa Llop, estos métodos pueden ser adaptados al ámbito del diseño: "Durante los últimos 50 años, los diseñadores hemos estructurado el proceso de un proyecto distribuyendo cada una de sus partes en forma de cascada. Primero definimos los requerimientos del encargo, los analizamos y sintetizamos, planteamos hipótesis, las valoramos, las implementamos y finalmente las entregamos a producción"6. Según Rosa Llop, el planteamiento de la metodología proyectual se suele ver como una secuencia lineal", algo que los diseñadores sabemos bien que no es posible. La práctica del diseño nos dice que la naturaleza del diseño tiene un carácter iterativo y una forma más cercana a una espiral evolutiva que a una escalera"7. Por lo tanto el proceso proyectual no es lineal, más bien funciona como una estructura cíclica que se comprueba constantemente, de manera que las diferentes partes de un proyecto pueden iniciarse al mismo tiempo y verificarse a través de sus diferentes estadios sin tener que esperar a que las fases previas concluyan. Desde este enfoque podemos entender el proceso de diseño como un proceso continuo, ya que nos permite evaluar cada una de las partes mientras este evoluciona. Incluso cuando el proyecto ha finalizado puede ser revisado en función de los resultados obtenidos, analizando los posibles errores cometidos y viendo de qué manera se podrían haber mejorado.

Anthony Dunne y Fiona Raby a través de las prácticas especulativas y el diseño crítico han generado alternativas que rompen con las lógicas de proceso proyectual. Ellos parten de dos concepciones o maneras de entender el diseño: el diseño afirmativo (en el que se fundamentan las prácticas del diseño convencional) y el diseño crítico. El diseño afirmativo se basa en el ahora como única posibilidad para dar solución a un problema de diseño, mientras que el diseño crítico tiene en cuenta otras posibles alternativas (posible, plausible, probable o preferible). Desde el enfoque crítico el diseñador ya no se dedica a resolver problemas (problem solving), sino a detectarlos (problem finding) ${ }^{8}$. Si el diseño ha estado tradicionalmente al servicio de la industria, ellos proponen que debe estar al servicio de la sociedad, donde las personas ya no son consumidores sino ciudadanos y la actitud del diseñador ya no se centra en dar respuestas sino en hacer preguntas ${ }^{9}$. De su enfoque teórico podemos deducir que e valor del diseño ya no reside en los objetos y comunicados producidos, sino en su relación con el entorno y el contexto; en el proceso de diseñar y en la experiencia de los agentes involucrados en el mismo.

\section{Diseñar desde el contexto}

Annelys de Vet es diseñadora gráfica y miembro de departamento del diseño del Sandberg Instituut Amsterdam (Masters Rietveld Academie $)^{10}$. De Vet considera que el diseño tiende a operar como un medio cuyo valor se sitúa en el proceso de diseñar. En 2007 publicó Subjective Atlas of Palestine, un libro que pretende reflexionar sobre la comunidad palestina. Durante el proyecto Annelys de Vet entrevistó a diseñadores, artistas y estudiantes para que representarán su visión de Palestina basándose en intereses personales y experiencias diarias, para tratar de dibujar una imagen de su humanidad desde dentro hacia fuera"1". Durante el proyecto los estudiantes tuvieron dificultades para poder definir su identidad vinculada al lugar. Esto se debe al constante cambio al que se ha visto sometido el territorio palestino. El proceso de trabajo de Subjective Atlas of Palestine, sirvió como catalizador para los estudiantes que investigaron para poder llegar a entender la sociedad palestina contemporánea. El resultado del proyecto, que tuvo como uno de sus objetivos la creación de un libro, recoge el imaginario creado por los participantes proyectando una imagen diferente de la sociedad palestina. Pero ¿qué repercusión pueden tener estas imágenes frente a la imagen proyectada por los medios de comunicación?, y ¿de qué manera afectan estas imágenes en nuestra percepción sobre la comunidad palestina?. Si el objeto de diseño como medio de comunicación queda anulado por la información hegemónica proyectada por los medios de comunicación, es más que evidente que su fin comunicador no conseguirá modificar nuestra visión sobre la sociedad. Para De Vet, lo realmente importante del proyecto y su proceso, es la CHRISTINA (2013). Share This 9 DUNNE, ANTHONY; RABY, FIONA (2013). Speculative everything. Cambridge: MIT Procial dreaming.

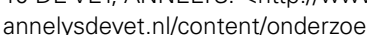
biography/biography AnnelysdeVet. htm > Iconsulta: 05/06/15 I1 DEVET, ANNELYS. Designing design approaches. <http://www. bureaudevet.be/articles/designing-against-populism-or-redefning-design-approaches/> lconsulta: 05/06/15 
experiencia que tienen las personas implicadas en el mismo, ya que el trabajo realizado puede generar más significado que la historia que se cuenta. Desde esta perspectiva, el diseño del libro puede ser visto como una forma de abrir un diálogo en relación al problema de Palestina y no como un producto final de diseño; el valor del proceso, como advertimos anteriormente, está en el proceso de creación del libro, cuyos resultados, experiencias y objetivos no se pueden predecir a través de los procesos y métodos del diseño. El proceso de diseño ya no es un fin en sí mismo, sino un medio que permite modificar la conductas de las personas. La experiencia en cada comunidad es distinta, no sólo por la visión de los participantes en el proyecto, sino por la forma de abordar el mismo y las relaciones que se establecen entre ellos.

En Subjective Atlas of Palestine hay otro factor importante a tener en cuenta: la difusión del imaginario colectivo; el producto final: el libro, se ve sometido a un continuo cambio ya que su distribución es gratuita y permite la reutilización de las imágenes para poder generar otros significados diferentes a los que fueron creados. Hablamos pues de la comunicación frente a la comunicación, de la forma en que se anulan nuestros mensajes emitidos como comunicadores. Por lo tanto, el diseño puede ser entendido no sólo como un instrumento que organiza determinada información en un contexto, sino como una alternativa para hacer frente a la información ${ }^{12}$ El proceso de "diseñar" es una experiencia que adquiere su valor en la transmisión de una nueva forma de entender la disciplina; la experiencia de los diseñadores y su forma de abordar los problemas de sociedades en conflicto prepara a los diseñadores para entender mejor el contexto sociocultural del problema a resolver. Lo realmente importante es el propósito del diseñador frente a la propuesta definitiva de la solución a un determinado problema de comunicación ${ }^{13}$. La experiencia y el análisis del contexto se traduce en una forma de hacer $y$ de pensar. Trabajar desde el contexto implica obtener información que de otra forma nos sería imposible conseguir esta información se traduce en las vivencias y experiencias de trabajar con la comunidad o grupo de personas de donde surge el problema. Este modo de practicar el diseño no puede ser abordado por metodologías proyectuales basadas en la experiencia previa. No existe un orden lógico para abordar el problema ya que este se define plenamente en el contexto.

Sheila Levrant de Bretteville ya en la década de 1970 entendió el diseño como un medio para trabajar con la comunidad y detectar sus problemáticas, viendo de qué forma estas afectan a las personas y que circunstancias se daban en el contexto. A través de la investigación localizaban grupos y organizaciones que estuvieran trabajando para dar solución a estos problemas, estudiaban su trabajo y detectaban necesidades que todavía no estaban siendo resueltas. Por medio del diseño establecían una comunicación con aquellas audiencias que no tenían información sobre estos problemas. La traducción de estos valores relacionales al diseño son descritos por De Bretteville como un conjunto de estrategias de comunicación que toman en cuenta a la audiencia como una parte activa del proceso de comunicación, de esta

\section{Bibliografía}

BONSIEPE, GUI (1985). El diseño de la periferia. Barcelona: Gustavo de la periferia. Barcelona: Gustavo
Gili. crisis. Valencia: Campgràtic. BREUER, GERDA; MEER, JULIA 2012). Women in Graphic Design 1890-2012. Frauen und Grakif-Design. Berlín: Jovis. CHAVES, NORBERTO (2001). E oficio de diseñar: propuestas a comienzan. Barcelona: Editoria Gumienzan. Baili. DEL CARMEN VILCHIS, LUZ (2002). Metodología del diseño: fundamertos teóricos. México: UNAM. DE VET, ANNELYYS (2007). Subjec-
Dus tive atlas of Palestine. Rotterdam. 010 Publishers. DUNNE, ANTHONY; RABY, FIONA 2013). Speculative everything. design, fiction, and social dreaming. Cambridge: MIT Press.

MAZE, RAMIA; OLAUSSON, LISA JOHAN; ZETTERLUND, CHRISTINA (2013). Share This Book: Critical Perspectives and Dialogues About
Poritical Design and Sustainability. Stockholm: Axl Books. KRIER, SOPHIE; RUYG, MARJOLIJN (2010). I don't know where I'm going, but I want to be there: The Expanding Field of Graphic Design 1900-2020. Amsterdam: BS
Publishers.
MUNARI, Bruno: RODRIGUEZ, Carmen Artal (1983). ¿Cómo nace los objetos?. Barcelona: Editorial Gustavo Gili.

MUNARI, BRUNO (1980). Diseño comunicación visual: contribución una metodología didáctica. Barcelona: Editorial Gustavo Gili. MUNARI, BRUNO (1968). El arte como oficio. Barcelona: Editorial Labor. W.AA. (2011). DESIGN ACT: Socially and Politically Engaged Design Taday-Critical Roles and Emerging W.AA. (2009) laspis Forum on Design and Critical Practice: The Reader. Berlín: Sternberg Press. forma se implica al receptor y se pone en valor su opinión sobre el problema. Estas estrategias también permiten comunicar desde múltiples perspectivas y enviar mensajes contradictorios a través de imágenes y textos, de manera que el receptor adopta una posición crítica ante e mensaje. Este modelo de comunicación, en el que imágenes y textos se yuxtaponen en diferentes niveles de lectura, pueden ser considerados como un desafío político ya que obliga al espectador a tomar una decisión sobre la construcción del significado.

\section{Educar a través del diseño}

El enfoque educativo del programa "Media Design Practices" en el Art Center College of Design en Pasadena, California, del que es directora Anne Burdick, consiste en preparar a los diseñadores para que "piensen" y "construyan" el mundo en base a sus experiencias personales, tratando de amplificar el significado del diseño. El programa educativo se basa en el trabajo en el contexto, utilizando el diseño para hacer preguntas por medio de proyectos aplicados y especulativos. Según Anne Burdick, los diseñadores van más allá del paradigma de resolución de problemas para situar al diseño como un medio que les permite entender y relacionarse con el mundo. El programa cuenta con un itinerario Field en colaboración con Designmatters ${ }^{14}$, en el que los estudiantes trabajan en un contexto sociocultural real para poder tomar conciencia de los problemas éticos, políticos y económicos que implica trabajar como diseñadores. El eje principal de la educación gira entorno a la hibridación disciplinaria y cultural, y en una fuerte reflexión crítica desde donde el diseño se practica con un claro compromiso social. Anne sostiene que la disciplina del diseño ya no se pueden definir por su modo de producción (tipografía, diseño web, ilustración, etc.) lo que provoca una ruptura con la noción más academicista del diseño ${ }^{15}$. “El diseño como paradigma de investigación, el diseño como modo de aprendizaje, el diseño como mentalidad y el diseño como alfabetización. Tengo la esperanza de que estos marcos permitan a los diseñadores ver y entender el diseño más allá de su aplicación comercial" 16

Si el diseño ya no se define por su modelo de producción, ¿no deberíamos replantearnos los procesos, métodos y metodologías en los que se basa?. Ya no se trata de ofrecer soluciones únicas como resultado de nuestro trabajo, se trata de tener experiencias en el contexto a través del diseño y en colaboración con otras personas y ámbitos de conocimiento, utilizando el diseño como un diálogo abie to ${ }^{17}$. El diseño debe relacionarse con la sociedad para escapar de los principios económicos que rigen nuestro mundo global; sus objetivos deben dar respuesta a los nuevas problemáticas que surgen de las sociedades cada vez más complejas. Además, el diseño debe ser un medio social que nos permita escapar del discurso hegemónico de los medios de comunicación y los modelos de producción. Para ello, debemos repensar el diseño, así como los procesos, métodos y metodologías que este utiliza. 